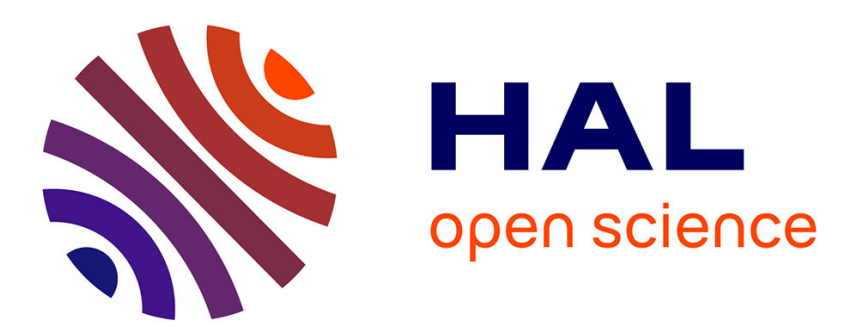

\title{
Maximum principle and bang-bang property of time optimal controls for Schrödinger type systems
}

Jérôme Lohéac, Marius Tucsnak

\section{To cite this version:}

Jérôme Lohéac, Marius Tucsnak. Maximum principle and bang-bang property of time optimal controls for Schrödinger type systems. SIAM Journal on Control and Optimization, 2013, 51 (5), pp.4016-4038. $10.1137 / 120872437$. hal-00685359v3

\section{HAL Id: hal-00685359 \\ https://hal.science/hal-00685359v3}

Submitted on 16 Apr 2013

HAL is a multi-disciplinary open access archive for the deposit and dissemination of scientific research documents, whether they are published or not. The documents may come from teaching and research institutions in France or abroad, or from public or private research centers.
L'archive ouverte pluridisciplinaire HAL, est destinée au dépôt et à la diffusion de documents scientifiques de niveau recherche, publiés ou non, émanant des établissements d'enseignement et de recherche français ou étrangers, des laboratoires publics ou privés. 


\title{
Maximum principle and bang-bang property of time optimal controls for Schrödinger type systems
}

\author{
Jérôme Lohéac* Marius Tucsnak*†
}

April 16, 2013

\begin{abstract}
We consider the time optimal control problem, with a point target, for a class of infinite dimensional systems with a dynamics governed by an abstract Schrödinger type equation. The main results establish a Pontryagyn type maximum principle and give sufficient conditions for the bangbang property of optimal controls. The results are then applied to some systems governed by partial differential equations. The paper ends by a discussion of possible extensions and by stating some open problems.
\end{abstract}

Key words. time optimal control, Pontryagyn's maximum principle, Bang-Bang property, Schrödinger equation

\section{Introduction}

Time optimal control is a classical problem for linear finite dimensional systems. For theses systems, among the interesting properties of time optimal controls, the facts that they satisfy Pontryagyn's maximum principle and that they are bang-bang, are well-known (see Bellman, Glicksberg and Gross [4]). The first extensions of these results to infinite dimensional linear systems have been given in Fattorini's paper [7]. The progress made in this field has been successively reported in the books of Lions [20] and of Fattorini [9]. In particular, the bang-bang property of time optimal controls has been quite rapidly established for invertible input operators. In the case of systems governed by PDE's, this means, roughly speaking, that the control is active in the entire spatial domain where the PDE is considered. The situation in which the control is active only in a part of the considered domain (or of its boundary) has been considered only recently. The results in this direction are limited as far as we know, to systems governed by linear parabolic equations (see, for instance, Mizel and Seidman [23], Wang [30], Phung and Wang [25], Kunisch and Wang [17]). The methodology used in most of the above mentioned works, based on a special $L^{\infty}$ null-controllability property of the considered system, does not seem applicable to systems which are time reversible, as those governed by wave, Schrödinger or Euler Bernoulli equations. The first aim of this paper is to show that for some of these systems, provided that they are exactly controllable in arbitrarily small time, Pontryagyn's maximum principle holds in a standard form. Moreover, assuming that the system is approximatively controllable with controls active only for times $t$ in a set of positive measure, we prove that time optimal controls have

\footnotetext{
${ }^{*}$ E-mail: jerome.loheac@univ-lorraine.fr and marius.tucsnak@univ-lorraine.fr

${ }^{\dagger}$ Both authors are with Institut Élie Cartan UMR 7502, Nancy-Université, CNRS, INRIA, B.P. 239, F-54506 Vandoeuvre-lès-Nancy Cedex, France, and INRIA Nancy Grand Est, Projet CORIDA.
} 
the bang-bang property and they are unique. We provide several examples of applications of these results to systems governed by the Schrödinger or the Euler-Bernoulli plate equations.

Let us recall, in order to give the precise statement of our main result, some classical notation and definitions from control theory.

We first introduce some notation. Let $X$ and $Y$ be Hilbert spaces. If $P \in \mathcal{L}(X ; Y)$ then the null-space and the range of $P$ are the subspaces of $X$ and $Y$ respectively defined by

$$
\text { Ker } P=\{x \in X, P x=0\}, \quad \text { Ran } P=\{P x, x \in X\} .
$$

Throughout this paper, $X$ and $U$ are complex Hilbert spaces, identified with their duals. The inner product and the norm in $X$ are denoted by $\langle\cdot, \cdot\rangle_{X}$ and $\|\cdot\|_{X}$, respectively. We denote by $\mathbb{T}=\left(\mathbb{T}_{t}\right)_{t \geqslant 0}$ a strongly continuous semigroup on $X$ generated by an operator $A: \mathcal{D}(A) \rightarrow X$.

Let $B \in \mathcal{L}(U, X)$ be a control operator, let $z_{0} \in X$ and let $u \in L^{2}([0, \infty), U)$. We consider the infinite dimensional system described by the equation

$$
\dot{z}(t)=A z(t)+B u(t), \quad z(0)=z_{0} .
$$

With the above notation, the solution $z$ of (1.1) is defined by

$$
z(t)=\mathbb{T}_{t} z_{0}+\Phi_{t} u \quad(t \geqslant 0),
$$

where $\Phi_{t} \in \mathcal{L}\left(L^{2}([0, t], U) ; X\right)$ is given by

$$
\Phi_{t} u=\int_{0}^{t} \mathbb{T}_{t-\sigma} B u(\sigma) \mathrm{d} \sigma \quad\left(u \in L^{2}([0, \infty), U)\right) .
$$

The maps $\left(\Phi_{t}\right)$ are called input to state maps.

Recall the following classical definitions (see, for instance, Tucsnak and Weiss [29, Sections 4.2 and 11.1]):

\section{Definition 1.1.}

- The pair $(A, B)$ is said approximatively controllable in time $\tau$ if $\operatorname{Ran} \Phi_{\tau}$ is dense in $X$.

- The pair $(A, B)$ is exactly controllable in time $\tau$ if $\operatorname{Ran} \Phi_{\tau}=X$.

We also need the following (less classical) definition.

Definition 1.2. Let $e \subset[0, \tau]$ be a set of positive Lebesgue measure. The pair $(A, B)$ is said approximatively controllable in time $\tau$ from $e$ if the range of the map $\Phi_{\tau, e} \in \mathcal{L}\left(L^{2}([0, \tau], U), X\right)$ defined by

$$
\Phi_{\tau, e} u=\int_{e} \mathbb{T}_{\tau-\sigma} B u(\sigma) \mathrm{d} \sigma \quad\left(u \in L^{2}([0, \tau], U)\right)
$$

is dense in $X$.

We are now in position to give a precise definition of time optimal controls.

Definition 1.3. Let $z_{0}, z_{1} \in X$ with $z_{0} \neq z_{1}$. A function $u^{*} \in L^{\infty}([0, \infty), U)$ is said a time optimal control for the pair $(A, B)$, associated to the initial state $z_{0}$ and the final state $z_{1}$, if there exists $\tau^{*}>0$ such that

1. $z_{1}=\mathbb{T}_{\tau^{*}} z_{0}+\Phi_{\tau^{*}} u^{*}$ and $\left\|u^{*}\right\|_{L^{\infty}\left(\left[0, \tau^{*}\right], U\right)} \leqslant 1 ;$ 
2. If $\tau>0$ is such that there exists $u \in L^{\infty}([0, \tau], U)$ with

$$
z_{1}=\mathbb{T}_{\tau} z_{0}+\Phi_{\tau} u, \quad\|u\|_{L^{\infty}([0, \tau], U)} \leqslant 1
$$

then $\tau \geqslant \tau^{*}$.

Our first main result provides a class of infinite dimensional system for which the maximum principle from the linear finite dimensional case can be extended in its classical form.

Theorem 1.4. Suppose that $(A, B)$ is exactly controllable in any time. Moreover, assume that $z_{0}, z_{1} \in$ $X, z_{0} \neq z_{1}$ are such that there exist $\tau>0$ and $u \in L^{\infty}([0, \tau], U)$ such that (1.4) holds. Then there exists a time optimal control $u^{*}$ steering $z_{0}$ to $z_{1}$ in time $\tau^{*}=\tau^{*}\left(z_{0}, z_{1}\right)$. Moreover, there exists $\eta \in X$, $\eta \neq 0$, such that

$$
\operatorname{Re}\left\langle B^{*} \mathbb{T}_{\tau^{*}-t}^{*} \eta, u^{*}(t)\right\rangle_{U}=\max _{\substack{\mathrm{v} \in U,\|\mathrm{v}\|_{U} \leqslant 1}} \operatorname{Re}\left\langle B^{*} \mathbb{T}_{\tau^{*}-t}^{*} \eta, \mathrm{v}\right\rangle_{U} \quad\left(t \in\left(0, \tau^{*}\right) \quad \text { a.e. }\right) .
$$

Remark 1.5. In the case of a skew-adjoint generator $A$, as shown in Proposition 2.2 below, the second assumption in the above theorem (i.e., the existence of $\tau>0$ and of $u \in L^{\infty}([0, \tau], U)$ such that (1.4) holds) follows from the first one, i.e., from the exact controllability of the pair $(A, B)$.

Remark 1.6. Assuming again that $X$ is infinite dimensional and $A$ is skew-adjoint, the assumptions of Theorem 1.4 cannot hold with a finite dimensional input space $U$. Indeed, the facts that $(A, B)$ is exactly controllable and $B \in \mathcal{L}(U, X)$ imply that $A-B B^{*}$ generates an exponentially stable semigroup(see, for instance, [21]). On the other hand, assuming by absurd that $U$ is finite dimensional implies that $A-B B^{*}$ is a compact perturbation of $A$, which generates an unitary group. Or according to Theorem 3 in Guo, Guo and Zhang [10], this fact cannot hold under our assumptions, so we have obtained a contradiction.

Our second main result shows that, under an extra assumption, the time optimal controls in the above theorem are bang-bang.

Corollary 1.7. With the notation and the assumptions in Theorem 1.4, assume moreover that the pair $(A, B)$ is approximatively controllable in time $\tau^{*}$ from any $e \subset\left[0, \tau^{*}\right]$ of positive measure. Then the time optimal control $u^{*}$ is bang-bang, in the sense that

$$
\left\|u^{*}(t)\right\|_{U}=1 \quad\left(t \in\left[0, \tau^{*}\right] \quad \text { a.e. }\right) .
$$

Moreover, the time optimal control is unique.

In some situations in finite dimensional theory or for parabolic PDE's, one can take $U=\mathbb{R}$. The constraint on the control considered above simply writes

$$
|u(t)| \leqslant 1 \quad(t \geqslant 0 \quad \text { a.e. })
$$

The bang-bang character of the time optimal control $u^{*}$ simply means that $u^{*}(t) \in\{-1,1\}$ for almost every $t>0$. In this situation an important role in computing time optimal controls is played by the switching locus which is formed by those instants $t \in\left[0, \tau^{*}\right]$ when $u^{*}$ is discontinuous (see, for instance, Lee and Markus [19, Section 2.5]). The problem of switching locus does not, at least as stated above, make sense in the general framework considered in Theorem 1.4. Indeed, according to Remark 1.6, our assumptions exclude the case of an finite dimensional input space $U$.

A situation in which the exact controllability holds with a finite dimensional input space and for which the question of switching locus make sense is described in Section 5 where we consider an example of system with unbounded input operator with a larger class of constraints on the control. 


\section{Some background on infinite dimensional systems}

In this section we gather, for easy reference, some results on infinite dimensional systems with bounded input and observation operators.

We continue to use the notation introduced in the previous section. In particular, $X, U$ and $Y$ are Hilbert spaces, $A: \mathcal{D}(A) \rightarrow X$ is the generator of a $C^{0}$ semigroup $\mathbb{T}$ in $X, B \in \mathcal{L}(U, X)$ is a control operator and $C \in \mathcal{L}(X, Y)$ is an observation operator.

The concept of exact controllability in Definition 1.1 says that we can reach any final state by means of controls in $L^{2}([0, \tau], U)$. The usual statement of time optimal control problems considers controls in $L^{\infty}([0, \tau], U)$. This difference is not essential in the case of bounded input operators as shown in the result below.

Proposition 2.1. Let $\tau>0$ and let

$$
R_{\tau}=\int_{0}^{\tau} \mathbb{T}_{t} B B^{*} \mathbb{T}_{t}^{*} \mathrm{~d} t \in \mathcal{L}(X),
$$

be the controllability Gramian in time $\tau$ for the pair $(A, B)$. Assume that the pair $(A, B)$ is exactly controllable in time $\tau$. Then $R_{\tau}$ is invertible and, for every $z_{0}, z_{1} \in X$, the control $u \in C([0, \tau], X)$ defined by

$$
u(t)=B^{*} \mathbb{T}_{\tau-t}^{*} R_{\tau}^{-1}\left(z_{1}-\mathbb{T}_{\tau} z_{0}\right) \quad(t \in[0, \tau]),
$$

steers the solution $z$ of (1.1) to $z_{1}$ in time $\tau$.

For a proof of this result we refer, for instance, to Zabczyk [31, Theorem 2.3, p. 210].

In the case of skew-adjoint generators, the above result implies a global controllability property under constraints. More precisely, we have the following result, which is essentially contained in Phung, Wang, Zhang [26]. However, for the sake of completeness, we give a relatively short proof below.

Proposition 2.2. With the notation and hypothesis in Proposition 2.1, assume that $A$ is skew-adjoint. Then for every $z_{0}, z_{1} \in X$ there exists $T>0$ and

$$
u \in L^{\infty}([0, T], U), \quad\|u\|_{L^{\infty}([0, T], U)} \leqslant 1,
$$

such that the solution $z$ of (1.1) satisfies the condition $z(T)=z_{1}$.

In order to prove the above proposition, we need the following result. We give here a direct proof. We refer to [26] for a duality based proof of a more general result.

Lemma 2.3. With the notation and assumptions in Proposition 2.2, take $M>0$. Then there exists $T>0$, depending only on $M$ and on the operators $A$ and $B$, such that for every $z_{0} \in X$ with $\left\|z_{0}\right\| \leqslant M$ one can find

$$
u \in L^{\infty}([0, T], U), \quad\|u\|_{L^{\infty}([0, T], U)} \leqslant 1,
$$

such that the solution $z$ of (1.1) satisfies the condition $z(T)=0$.

Proof. Using the fact that $\mathbb{T}$ is in this case an unitary group, together with (2.1), it follows that any point in the closed ball of center $\mathbb{T}_{\tau} z_{0}$ and of radius $\left\|B^{*}\right\|_{\mathcal{L}(X, U)}^{-1}\left\|R_{\tau}^{-1}\right\|_{\mathcal{L}(X)}^{-1}$ can be reached by a control such that $\|u\|_{L^{\infty}([0, \tau], U)} \leqslant 1$. This means, in particular, that the conclusion of the lemma holds (with 
$T=\tau)$ for $M \leqslant\left\|B^{*}\right\|_{\mathcal{L}(X, U)}^{-1}\left\|R_{\tau}^{-1}\right\|_{\mathcal{L}(X)}^{-1}$. Therefore, without loss of generality, we can assume that $M>\left\|B^{*}\right\|_{\mathcal{L}(X, U)}^{-1}\left\|R_{\tau}^{-1}\right\|_{\mathcal{L}(X)}^{-1}$ and it suffices to prove the result for $z_{0} \in X$ with

$$
\left\|B^{*}\right\|_{\mathcal{L}(X, U)}^{-1}\left\|R_{\tau}^{-1}\right\|_{\mathcal{L}(X)}^{-1}<\left\|z_{0}\right\| \leqslant M
$$

For such $z_{0}$ we define $\alpha_{1} \in X$ by

$$
\alpha_{1}=\left(1-\frac{\left\|B^{*}\right\|_{\mathcal{L}(X, U)}^{-1}\left\|R_{\tau}^{-1}\right\|_{\mathcal{L}(X)}^{-1}}{2\left\|z_{0}\right\|}\right) \mathbb{T}_{\tau} z_{0} .
$$

Since we clearly have $\left\|\alpha_{1}-\mathbb{T}_{\tau} z_{0}\right\| \leqslant\left\|B^{*}\right\|_{\mathcal{L}(X, U)}^{-1}\left\|R_{\tau}^{-1}\right\|_{\mathcal{L}(X)}^{-1}$, it follows that there exists $u \in L^{\infty}([0, \tau], U)$, $\|u(t)\| \leqslant 1$ a.e. such that the solution $z$ of $(1.1)$ satisfies the condition $z(\tau)=\alpha_{1}$. Moreover, from (2.3) it easily follows that

$$
\left\|\alpha_{1}\right\|_{X} \leqslant M-\frac{1}{2}\left\|B^{*}\right\|_{\mathcal{L}(X, U)}^{-1}\left\|R_{\tau}^{-1}\right\|_{\mathcal{L}(X)}^{-1} .
$$

Let $n$ be the largest integer such that

$$
\frac{n}{2}\left\|B^{*}\right\|_{\mathcal{L}(X, U)}^{-1}\left\|R_{\tau}^{-1}\right\|_{\mathcal{L}(X)}^{-1} \leqslant M .
$$

Iterating the above argument on the intervals $[k \tau,(k+1) \tau], k \in \mathbb{N}$, it follows that there exists

$$
\alpha_{n} \in X, \quad\left\|\alpha_{n}\right\|_{X} \leqslant M-\frac{n}{2}\left\|B^{*}\right\|_{\mathcal{L}(X, U)}^{-1}\left\|R_{\tau}^{-1}\right\|_{\mathcal{L}(X)}^{-1}<\left\|B^{*}\right\|_{\mathcal{L}(X, U)}^{-1}\left\|R_{\tau}^{-1}\right\|_{\mathcal{L}(X)}^{-1},
$$

and

$$
u \in L^{\infty}([0, n \tau], U), \quad\|u\|_{L^{\infty}([0, n \tau], U)} \leqslant 1,
$$

such that $z(n \tau)=\alpha_{n}$. Using again the fact that $\mathbb{T}$ is in this case an unitary group, together with (2.1) it follows that we can steer $\alpha_{n}$ to zero in time $\tau$ by means of controls with $\|u(t)\| \leqslant 1$. Since $n$ depends only on $M$ and the operators $A$ and $B$, we have thus shown that the conclusion of the lemma holds with $T=(n+1) \tau$.

We next give, following [26], the proof of Proposition 2.2.

Proof of Proposition 2.2. Applying this result with $M=2\left(\left\|z_{0}\right\|_{X}+\left\|z_{1}\right\|_{X}\right)$ it follows that there exists $T>0$ (depending only on $M$ ) and $u$ satisfying (2.2) such that the solution $\xi$ of

$$
\dot{\xi}(t)=A \xi(t)+B u(t), \quad \xi(0)=z_{0}-\mathbb{T}_{T}^{-1} z_{1},
$$

satisfies $\xi(T)=0$. Setting

$$
z(t)=\xi(t)+\mathbb{T}_{t} \mathbb{T}_{T}^{-1} z_{1} \quad(t \in[0, T]),
$$

it is easily seen that we have

$$
\dot{z}(t)=A z(t)+B u(t) \quad(t \in[0, T]),
$$

together with $z(0)=z_{0}$ and $z(T)=z_{1}$, which concludes the proof.

Definition 2.4. The $L^{\infty}$ reachable space at time $t$ of the pair $(A, B)$ is defined as

$$
R_{t}^{\infty}=\Phi_{t}\left(L^{\infty}([0, t], U)\right) .
$$


By a slight variation of the argument in [9, Lemma 2.1.1] one can check that, for each $t>0$, when endowed with the norm

$$
\|x\|_{R_{t}^{\infty}}=\inf \left\{\|u\|_{L^{\infty}([0, t], U)}, \Phi_{t} u=x\right\} \quad(x \in X),
$$

$R_{t}^{\infty}$ is a Banach space.

Proposition 2.5. Let $0 \leqslant \sigma \leqslant t$. Then we have the continuous inclusions

$$
R_{\sigma}^{\infty} \subset R_{t}^{\infty} \subset X .
$$

Proof. It is clear that the above inclusions hold. To show that they are continuous, we take $x \in R_{t}^{\infty}$, so that there exists $u \in L^{\infty}([0, t], U)$ with $x=\Phi_{t} u$. Since $\Phi_{t} \in \mathcal{L}\left(L^{2}([0, t], U), X\right)$ it follows that there exists $M>0$ such that $\|x\|_{X} \leqslant M\|u\|_{L^{\infty}([0, t], U)}$ for every $u \in L^{\infty}([0, t], U)$ satisfying $\Phi_{t} u=x$. Consequently, we have

$$
\|x\|_{X} \leqslant M \inf _{\substack{u \in L^{\infty}([0, t], U) \\ \Phi_{t} u=x}}\|u\|_{L^{\infty}([0, t], U)}=M\|x\|_{R_{t}^{\infty}}
$$

so that the inclusion $R_{t}^{\infty} \subset X$ is continuous.

To prove the continuity of the inclusion $R_{\sigma}^{\infty} \subset R_{t}^{\infty}$, it suffices to note that if $x \in R_{\sigma}^{\infty}$ and $u \in L^{\infty}([0, \sigma], U)$ are such that $x=\Phi_{\sigma} u$ then $\tilde{u} \in L^{\infty}([0, t], U)$ defined for almost every $s \in[0, t]$ by $\tilde{u}(s)=\chi_{(t-\sigma, t)}(s) u(s-t+\sigma)$, where $\chi_{(t-\sigma, t)}$ denotes the characteristic function of the set $(t-\sigma, t)$, has the property $x=\Phi_{t} \tilde{u}$ and $\|\tilde{u}\|_{L^{\infty}([0, t], U)}=\|u\|_{L^{\infty}([0, \sigma], U)}$.

From Proposition 2.1 it follows that if $(A, B)$ is exactly controllable in some time $\tau>0$ then $R_{\tau}^{\infty}=X$. This fact, combined with Proposition 2.5 and with the closed graph theorem clearly imply the result below.

Corollary 2.6. Assume that $(A, B)$ is exactly controllable in some time $\tau>0$. Then the norms $\|\cdot\|_{R_{\tau}^{\infty}}$ and $\|\cdot\|_{X}$ are equivalent.

Remark 2.7. If $R_{t}^{\infty}=X$, the control cost $C_{t}$ in time $t$ is defined by

$$
C_{t}=\sup _{x \neq 0} \frac{\|x\|_{R_{t}^{\infty}}}{\|x\|_{X}} \quad(t>0) .
$$

It is easily seen that the map $t \mapsto C_{t}$ is non-increasing.

We also need some concepts of observability theory. Let $Y$ be another Hilbert space and let $C \in \mathcal{L}(X, Y)$ be an observation operator for the semigroup $\mathbb{T}$. For $\tau>0$ we consider the initial state to output map $\Psi_{\tau}$ defined by

$$
\left(\Psi_{\tau} z_{0}\right)(t)=C \mathbb{T}_{t} z_{0} \quad\left(z_{0} \in X, t \in[0, \tau]\right) .
$$

The operators $\Psi_{\tau}$ are briefly called output maps corresponding to the pair $(A, C)$. If $e \subset[0, \tau]$ is a set of positive measure, we consider the restriction of the above defined initial state to output map to a set of positive measure $e \subset[0, \tau]$, which is defined by

$$
\Psi_{\tau, e} \in \mathcal{L}\left(X, L^{2}([0, \tau], Y)\right), \quad \Psi_{\tau, e}=\chi_{e} \Psi_{\tau},
$$

where $\chi_{e}$ is the characteristic function of $e$. 
We denote by $\Psi_{\tau}^{d}$ (respectively $\Psi_{\tau, e}^{d}$ ) the output maps corresponding (respectively the restriction to a set of positive measure $e \subset[0, \tau]$ of these output maps) corresponding to the pair $\left(A^{*}, B^{*}\right)$, i.e., we set

$$
\begin{aligned}
& \left(\Psi_{\tau}^{d} z_{0}\right)(t)=B^{*} \mathbb{T}_{t}^{*} z_{0} \quad\left(z_{0} \in X, t \in[0, \tau]\right), \\
& \Psi_{\tau, e}^{d} \in \mathcal{L}\left(X, L^{2}([0, \tau], U)\right), \quad \Psi_{\tau, e}^{d}=\chi_{e} \Psi_{\tau}^{d} .
\end{aligned}
$$

There are several generalizations of the concept of observability to infinite dimensional linear systems. In this paper we need only the following one.

Definition 2.8. Let $\tau>0$ and $e \subset[0, \tau]$ be a set of positive measure. The pair $(A, C)$ is said approximately observable from $e$ if $\operatorname{Ker} \Psi_{\tau, e}=\{0\}$.

We have the following duality result, which is a simple consequence of the corresponding result for $e=[0, \tau]$ (see, for instance [22, Proposition 2.4]).

Proposition 2.9. Let $\tau>0, e \subset[0, \tau]$ a set of positive measure and let

$$
e^{\prime}=\{\tau-t \quad \mid \quad t \in e\} .
$$

Then

$$
\Phi_{\tau, e}=\left(\Psi_{\tau, e^{\prime}}^{d}\right)^{*} \boldsymbol{Я}_{\tau},
$$

where $\left(\Psi_{\tau, e^{\prime}}^{d}\right)^{*} \in \mathcal{L}\left(L^{\infty}([0, \tau] ; U), X\right)$ is the dual operator of $\Psi_{\tau, e^{\prime}}^{d}$ and $\boldsymbol{Я}_{\tau}$ is the reflection operator on $L^{2}([0, \tau] ; U)$, defined by $\boldsymbol{Я}_{\tau} u(t)=u(\tau-t)$ (Notice that $\boldsymbol{Я}_{\tau}$ is self-adjoint and also unitary.).

In particular, the pair $(A, B)$ is approximatively controllable in time $\tau$ from a set of positive measure $e \subset[0, \tau]$ if and only if

$$
\operatorname{Ker} \Psi_{\tau, e^{\prime}}^{d}=\{0\} .
$$

Remark 2.10. If $e=[0, \tau]$ then formula (2.8) simply writes

$$
\Phi_{\tau}=\left(\Psi_{\tau}^{d}\right)^{*} \boldsymbol{Я}_{\tau} .
$$

\section{Main results}

In this section we extend the maximum principle, well-known for finite dimensional systems, to a class of infinite dimensional systems. To this aim, we continue to use all the notation in Section 2. In particular we denote by $X$ the state space (assumed to be Hilbert), $A$ stands for the semigroup generator and the control operator (assumed to be bounded) is still denoted by $B$. We also continue to use the notation $R_{t}^{\infty}$, introduced in (2.4), for the space which is reachable by $L^{\infty}$ input functions. Moreover, for every $t>0$, the closed unit ball in $R_{t}^{\infty}$ is:

$$
B_{t}^{\infty}(1)=\left\{\Phi_{t} u, u \in L^{\infty}([0, t], U),\|u\|_{L^{\infty}([0, t], U)} \leqslant 1\right\} .
$$

Before deriving the maximum principle, we note the following existence result for time optimal controls. We skip the proof of this result since it can be obtained by simple adaptation of the proofs in [9, Lemma 3.1.1 and Theorem 3.1.2].

Proposition 3.1. With the above notation and assumptions, assume moreover that $z_{0}, z_{1} \in X, z_{0} \neq z_{1}$ are such that there exists $t>0$ with

$$
z_{1}-\mathbb{T}_{t} z_{0} \in B_{t}^{\infty}(1) .
$$

Then there exists $\tau^{*}\left(z_{0}, z_{1}\right)>0$ such that

$$
\tau^{*}\left(z_{0}, z_{1}\right)=\min \left\{t>0 \quad \mid \quad z_{1}-\mathbb{T}_{t} z_{0} \in B_{t}^{\infty}(1)\right\}>0
$$


In other words $\tau^{*}$ is the minimal time in which $z_{0}$ can be steered to $z_{1}$ by controls satisfying $\|u(t)\| \leqslant 1$ for almost every $t$. Therefore, any $u^{*} \in L^{\infty}\left(\left[0, \tau^{*}\right], U\right)$ satisfying

$$
z_{1}-\mathbb{T}_{\tau^{*}} z_{0}=\Phi_{\tau^{*}} u^{*}
$$

is called a time optimal control for the pair $(A, B)$.

Let now give the proof of Theorem 1.4.

Proof of Theorem 1.4. The existence of a time optimal control $u^{*}$ is given by Proposition 3.1. To prove the maximum principle, we first show, in the spirit of [9], that $z_{1}-\mathbb{T}_{\tau^{*}} z_{0} \in \partial B_{\tau^{*}}^{\infty}(1)$, the boundary being taken in the sense of the topology of $R_{\tau^{*}}^{\infty}$ and hence, according to Corollary 2.6, in the sense of the topology of $X$. Assume, by contradiction, that $z_{1}-\mathbb{T}_{\tau^{*}} z_{0} \notin \partial B_{\tau^{*}}^{\infty}(1)$. This implies the existence of $r \in(0,1)$ such that $\frac{1}{r}\left(z_{1}-\mathbb{T}_{\tau^{*}} z_{0}\right) \in B_{\tau^{*}}^{\infty}(1)$, i.e., the existence of $u_{1} \in L^{\infty}\left(\left[0, \tau^{*}\right], U\right)$, $\left\|u_{1}\right\|_{L^{\infty}\left(\left[0, \tau^{*}\right], U\right)} \leqslant r<1$ such that $z_{1}-\mathbb{T}_{\tau^{*}} z_{0}=\Phi_{\tau^{*}} u_{1}$. Let $t<\tau^{*}$. Then

$$
\begin{aligned}
z_{1}-\mathbb{T}_{t} z_{0}=\mathbb{T}_{\tau^{*}} z_{0} & -\mathbb{T}_{t} z_{0}+\Phi_{\tau^{*}} u_{1}= \\
& =\Phi_{t} u_{1}+\mathbb{T}_{\tau^{*}} z_{0}-\mathbb{T}_{t} z_{0}+\int_{0}^{t}\left(\mathbb{T}_{\tau^{*}-\sigma}-\mathbb{T}_{t-\sigma}\right) B u_{1}(\sigma) \mathrm{d} \sigma+\int_{t}^{\tau^{*}} \mathbb{T}_{\tau^{*}-\sigma} B u_{1}(\sigma) \mathrm{d} \sigma .
\end{aligned}
$$

Let

$$
\varphi\left(t, \tau^{*}\right)=\mathbb{T}_{\tau^{*} z_{0}}-\mathbb{T}_{t} z_{0}+\int_{0}^{t}\left(\mathbb{T}_{\tau^{*}-\sigma}-\mathbb{T}_{t-\sigma}\right) B u_{1}(\sigma) \mathrm{d} \sigma+\int_{t}^{\tau^{*}} \mathbb{T}_{\tau^{*}-\sigma} B u_{1}(\sigma) \mathrm{d} \sigma \quad\left(t \in\left(0, \tau^{*}\right),\right.
$$

so that

$$
z_{1}-\mathbb{T}_{t} z_{0}=\Phi_{t} u_{1}+\varphi\left(t, \tau^{*}\right) .
$$

It is easily seen that $\lim _{t \rightarrow \tau^{*}} \varphi\left(t, \tau^{*}\right)=0$ in $X$. From Remark 2.7, we have that $\left\|\varphi\left(t, \tau^{*}\right)\right\|_{R_{t}^{\infty}} \leqslant$ $C_{t}\left\|\varphi\left(t, \tau^{*}\right)\right\|_{X}$, with $t \mapsto C_{t}$ a non-increasing function. This means that for $t$ close enough to $\tau^{*}$ there exists a control $u_{2} \in L^{\infty}([0, t], U)$ with

$$
\left\|u_{2}\right\|_{L^{\infty}([0, t], U)} \leqslant 1-r, \quad \Phi_{t} u_{2}=\varphi\left(t, \tau^{*}\right) .
$$

The above formula and (3.3) imply, denoting $u=u_{1}+u_{2}$, that

$$
z_{1}-\mathbb{T}_{t} z_{0}=\Phi_{t} u, \quad\|u\|_{L^{\infty}([0, t], U)} \leqslant 1
$$

which contradicts the fact that $\tau^{*}$ is minimal in the sense of (3.1). We have thus shown that indeed $z_{1}-\mathbb{T}_{\tau^{*}} z_{0} \in \partial B_{\tau^{*}}^{\infty}(1)$. This implies, using a consequence of the Hahn-Banach theorem (see, for instance, $\left[2\right.$, Theorem 1.13]) and the fact that the interior of $B_{\tau^{*}}^{\infty}(1)$ is non empty, that there exists $\eta \in X, \eta \neq 0$ such that

$$
\operatorname{Re}\left\langle\eta, z_{1}-\mathbb{T}_{\tau^{*}} z_{0}\right\rangle_{X} \geqslant \operatorname{Re}\langle\eta, x\rangle_{X} \quad\left(x \in B_{\tau^{*}}^{\infty}(1)\right) .
$$

The above formula, combined to the definition (3.2) of the time optimal control implies that

$$
\operatorname{Re} \int_{0}^{\tau^{*}}\left\langle B^{*} \mathbb{T}_{\tau^{*}-t}^{*} \eta, u^{*}(t)\right\rangle_{U} \mathrm{~d} t \geqslant \operatorname{Re} \int_{0}^{\tau^{*}}\left\langle B^{*} \mathbb{T}_{\tau^{*}-t}^{*} \eta, v(t)\right\rangle_{U} \mathrm{~d} t \quad\left(\|v\|_{L^{\infty}\left(\left[0, \tau^{*}\right], U\right)} \leqslant 1\right) .
$$

Using Lemma 2.2.1 of [9] and the fact that $t \mapsto B^{*} \mathbb{T}_{\tau^{*}-t}^{*} \eta$ is $U$-weakly measurable, we obtain that

$$
\sup _{\substack{v \in L^{\infty}\left(\left[0, \tau^{*}\right], U\right),\|v\|_{L^{\infty}\left(\left[0, \tau^{*}\right], U\right)} \leqslant 1}} \operatorname{Re} \int_{0}^{\tau^{*}}\left\langle B^{*} \mathbb{T}_{\tau^{*}-t}^{*} \eta, v(t)\right\rangle_{U} \mathrm{~d} t=\int_{0}^{\tau^{*}}\left\|B^{*} \mathbb{T}_{\tau^{*}-t}^{*} \eta\right\|_{U} \mathrm{~d} t,
$$


and hence we have

$$
\operatorname{Re} \int_{0}^{\tau^{*}}\left\langle B^{*} \mathbb{T}_{\tau^{*}-t}^{*} \eta, u^{*}(t)\right\rangle_{U} \mathrm{~d} t=\int_{0}^{\tau^{*}}\left\|B^{*} \mathbb{T}_{\tau^{*}-t}^{*} \eta\right\|_{U} \mathrm{~d} t
$$

On the other hand, we clearly have

$$
\operatorname{Re}\left\langle B^{*} \mathbb{T}_{\tau^{*}-t}^{*} \eta, u^{*}(t)\right\rangle_{U} \leqslant\left\|B^{*} \mathbb{T}_{\tau^{*}-t}^{*} \eta\right\|_{U} \quad\left(t \in\left[0, \tau^{*}\right] \quad \text { a.e. }\right)
$$

The last estimate and (3.4) imply that

$$
\operatorname{Re}\left\langle B^{*} \mathbb{T}_{\tau^{*}-t}^{*} \eta, u^{*}(t)\right\rangle_{U}=\left\|B^{*} \mathbb{T}_{\tau^{*}-t}^{*} \eta\right\|_{U} \quad\left(t \in\left[0, \tau^{*}\right] \quad \text { a.e. }\right),
$$

which implies (1.5).

We conclude this Section by giving the proof of Corollary 1.7.

Proof of Corollary 1.7. We know from Theorem 1.4 that there exists $\eta \in X, \eta \neq 0$, such that we have (1.5). On the other hand, the fact that the pair $(A, B)$ is approximatively controllable in time $\tau^{*}$ from any $e \subset\left[0, \tau^{*}\right]$ of positive measure, together with Proposition 2.9, ensures that $B^{*} \mathbb{T}_{\tau^{*}-t}^{*} \eta \neq 0$ for almost every $t \in\left[0, \tau^{*}\right]$. Therefore, (1.5) implies that the time optimal control is given by

$$
u^{*}(t)=\frac{1}{\left\|B^{*} \mathbb{T}_{\tau^{*}-t}^{*} \eta\right\|_{U}} B^{*} \mathbb{T}_{\tau^{*}-t}^{*} \eta \quad\left(t \in\left[0, \tau^{*}\right] \quad \text { a.e. }\right)
$$

which clearly implies (1.6).

The uniqueness of $u^{*}$ can be proved in a standard way, using the strict convexity of $U$ and the bangbang property of every time optimal control. Indeed, assume by contradiction that there exist two time optimal controls $u_{1}^{*} \neq u_{2}^{*}$. Then for $u^{*}=\frac{1}{2}\left(u_{1}^{*}+u_{2}^{*}\right) \in L^{\infty}\left(\left[0, \tau^{*}\right], U\right)$, we have $\left\|u^{*}\right\|_{L^{\infty}\left(\left[0, \tau^{*}\right], U\right)} \leqslant 1$, $\left\|u^{*}(t)\right\|_{U}<1$ for $t$ in some set of positive measure and $\Phi_{\tau^{*}} u^{*}=z_{1}-\mathbb{T}_{\tau^{*}} z_{0}$. Thus $u^{*}$ is a time optimal control which does not satisfy the bang-bang property, so that we have obtained a contradiction.

\section{Application to the Schrödinger equation}

We first give two preliminary lemmas for which, although the results seem quite simple, we did not find the exact statement in the literature.

The proof of these lemmas are obtained using a uniqueness theorem of Privalov (see, for instance, [32, Vol. II, ch. XIV, Theorem 1.9]), which says that if a holomorphic function in the unit disk has non tangential limits equal to zero on a set of positive measure of the unit circle then the function is equal to zero in the whole disk. The idea of using such lemmas for unique continuation of PDE's is borrowed from Reifler and Vogt [27] (see also Hante, Sigalotti and Tucsnak [11]).

Lemma 4.1. Let $I \subset \mathbb{Z},\left(\lambda_{n}\right)_{n \in I}$ be a sequence of real numbers bounded from below (or from above), let $e \subset \mathbb{R}$ a bounded set of positive measure and let $\left(a_{n}\right)_{n \in I} \in \ell^{1}(I, \mathbb{C})$. Assume that

$$
\sum_{n \in I} a_{n} e^{i \lambda_{n} t}=0 \quad(t \in e) .
$$

Then

$$
\sum_{n \in I} a_{n} e^{i \lambda_{n} t}=0 \quad(t \in \mathbb{R}) .
$$


Proof. Let

$$
\mathbb{C}_{+}=\{s \in \mathbb{C} \mid \operatorname{Im} s>0\}, \quad \mathbb{C}_{-}=\{s \in \mathbb{C} \mid \operatorname{Im} s<0\},
$$

and $\varphi$ be o conformal map of the unit disk $D$ of $\mathbb{C}$ to $\mathbb{C}_{+}$(respectively on $\mathbb{C}_{-}$). Let $g: D \rightarrow \mathbb{C}$ be defined by

$$
g(z)=\sum_{n \in I} a_{n} e^{i \lambda_{n} \varphi(z)} \quad(z \in D)
$$

Since $\left(a_{n}\right) \in \ell^{1}$ we have that $g$ is holomorphic in $D$ and continuous up to the boundary. Moreover, by (4.1) we have

$$
g\left(\varphi^{-1}(t)\right)=0 \quad(t \in e) .
$$

Using Privalov's uniqueness Theorem, [32, Vol. II, ch. XIV, Theorem 1.9] it follows that $g$ vanishes in $\bar{D}$, so that we obtain (4.2).

The assumption $\left(a_{n}\right) \in \ell^{1}$ in the above result is not appropriate some applications developed in Section 5, where the natural condition is $\left(a_{n}\right) \in \ell^{2}$. The result below shows that the conclusion of Lemma 4.1 still holds, provided that we do more assumptions on the sequence $\left(\lambda_{n}\right)$.

Lemma 4.2. Let $\left(a_{n}\right) \in \ell^{2}(\mathbb{N}, \mathbb{C})$ and let $\left(\lambda_{n}\right)$ be such that $\lambda_{n} \in \mathbb{N}$ for every $n \in \mathbb{N}$ and $\lambda_{n} \neq \lambda_{m}$ for $m \neq n$. Moreover, suppose that

$$
\sum_{n \in \mathbb{N}} a_{n} e^{i \lambda_{n} t}=0 \quad(t \in e),
$$

where $e \subset[0,2 \pi]$ is a set of positive measure. Then $a_{n}=0$ for every $n \in \mathbb{N}$.

Proof. Let $D$ be the open unit disk in $\mathbb{C}$ and let $g: D \rightarrow \mathbb{C}$ be defined by

$$
g(z)=\sum_{n \in I} a_{n} z^{\lambda_{n}} \quad(z \in D) .
$$

Since $\left(a_{n}\right) \in \ell^{2}$ we have that $g$ is holomorphic in $D$. Moreover, by Abel's theorem (see, for instance $[28$, p.229]), relation (4.3) implies that

$$
\lim _{\substack{z \rightarrow e^{i t} \\ \text { Ang }}} g(z)=0 \quad(t \in e)
$$

where the notation Ang means that $z \rightarrow e^{i t}$ non tangentially. Using Privalov uniqueness Theorem it follows that $g=0$ on $D$ and hence the result.

From Lemma 4.1, we can derive the following result:

Lemma 4.3. Let $X$ and $Y$ be Hilbert spaces, let $A_{0}$ be a self-adjoint and diagonalisable operator on $X$ with domain $\mathcal{D}\left(A_{0}\right)$ and let $C \in \mathcal{L}(X, Y)$. Moreover, assume that that the spectrum $\sigma\left(A_{0}\right)$ of $A_{0}$ satisfies $\sigma\left(A_{0}\right) \subset[m, \infty)$ for some $m \in \mathbb{R}$. Let $z_{0} \in X$ and $z \in C^{0}(\mathbb{R}, X), y \in C^{0}(\mathbb{R}, Y)$ satisfying

$$
\begin{gathered}
\dot{z}(t)=i A_{0} z(t) \quad(t \in \mathbb{R}), \quad z(0)=z_{0}, \\
y(t)=C z(t) \quad(t \in \mathbb{R}) .
\end{gathered}
$$

If

$$
y(t)=0 \quad(t \in e),
$$

for some set $e \subset \mathbb{R}$ of positive measure, then

$$
y(t)=0 \quad(t \in \mathbb{R}) .
$$


Proof. Since $A_{0}$ is assumed to be self-adjoint and diagonalisable, there exists an orthonormal basis of eigenvectors $\left(\varphi_{n}\right)_{n \in I}$ of $A_{0}$, with $I \subset \mathbb{Z}$ and a corresponding sequence of eigenvalues $\left(\lambda_{n}\right)_{n \in I}$, where $\left(\lambda_{n}\right)_{n \in I}$ is a sequence with values in $[m, \infty)$. The solution $z$ of $(4.4)$ writes

$$
z(t)=\sum_{n \in I} a_{n} e^{i \lambda_{n} t} \varphi_{n} \quad(t \in \mathbb{R}),
$$

where $a_{n}=\left\langle z_{0}, \varphi_{n}\right\rangle_{X}$ for every $n \in I$. Hence $y$ in (4.5) writes

$$
y(t)=\sum_{n \in I} a_{n} e^{i \lambda_{n} t} C \varphi_{n} \quad(t \in \mathbb{R}) .
$$

For any $\mathrm{v} \in Y$, we have

$$
\langle y(t), \mathrm{v}\rangle_{Y}=\sum_{n \in I} a_{n} e^{i \lambda_{n} t}\left\langle C \varphi_{n}, \mathrm{v}\right\rangle_{Y}=\sum_{n \in I} a_{n} e^{i \lambda_{n} t}\left\langle C^{*} \mathrm{v}, \varphi_{n}\right\rangle_{X} \quad(t \in \mathbb{R}) .
$$

Since $\left(a_{n}\right)_{n} \in \ell^{2}(I, \mathbb{C})$ and $\left(\left\langle C^{*} \mathrm{v}, \varphi_{n}\right\rangle_{X}\right)_{n} \in \ell^{2}(I, \mathbb{C})$, it follows that $\left(a_{n}\left\langle C^{*} \mathrm{v}, \varphi_{n}\right\rangle_{X}\right)_{n} \in \ell^{1}(I, \mathbb{C})$. Moreover, we know that $\langle y(t), \mathrm{v}\rangle_{Y}=0$ for every $t \in e$ and that the sequence $\left(\lambda_{n}\right)_{n \in I}$ is bounded from bellow, hence, using Lemma 4.1 , we deduce that $\langle y(t), \mathrm{v}\rangle_{Y}=0$ for every $t \in \mathbb{R}$. Since this property is true for every $\mathrm{v} \in Y$, we conclude that $y(t)=0$ for every $t \in \mathbb{R}$.

We next study a Schrödinger equation with distributed internal control. More precisely, we consider the system

$$
\begin{gathered}
\dot{z}(x, t)=-i \Delta z(x, t)+i a(x) z(x, t)+u(x, t) \chi_{\mathcal{O}}(x) \quad(x \in \Omega, \quad t \geqslant 0), \\
z(x, t)=0 \quad(x \in \partial \Omega, \quad t \geqslant 0),
\end{gathered}
$$

where $\Omega \subset \mathbb{R}^{n}$ is an open set, $\mathcal{O}$ is an open subset of $\Omega, \chi_{\mathcal{O}}$ is the characteristic function of $\mathcal{O}$ and the control $u$ is such that $\|u(\cdot, t)\|_{L^{2}(\mathcal{O})} \leqslant 1$ for almost every $t \geqslant 0$.

Proposition 4.4. Assume that one of the assumptions

1. The open set $\Omega$ is bounded, $\partial \Omega$ is of class $C^{3}, a \in L^{\infty}(\Omega, \mathbb{R})$ and $\mathcal{O}$ satisfies the geometric optics condition, as described in Bardos, Lebeau and Rauch [3];

2. The open set $\Omega$ is a rectangular domain, a is a real constant and $\mathcal{O}$ is an arbitrary nonempty open subset of $\Omega$,

holds. Then for every $z_{0}, z_{1} \in L^{2}(\Omega)$, with $z_{0} \neq z_{1}$, there exists a unique time optimal control $u^{*}$ steering the solution of (4.6)-(4.7) from $z_{0}(a t t=0)$ to $z_{1}\left(\right.$ at $\left.t=\tau^{*}=\tau^{*}\left(z_{0}, z_{1}\right)\right)$. Moreover, there exists $\eta \in L^{2}(\Omega), \eta \neq 0$, such that

$$
\operatorname{Re} \int_{\mathcal{O}} w(x, t) \overline{u^{*}}(x, t) \mathrm{d} x=\max _{\substack{\mathrm{v} \in L^{2}(\mathcal{O}),\|\mathrm{v}\|_{L^{2}(\mathcal{O})} \leqslant 1}} \operatorname{Re} \int_{\mathcal{O}} w(x, t) \overline{\mathrm{v}}(x) \mathrm{d} x \quad\left(t \in\left[0, \tau^{*}\right] \quad \text { a.e. }\right),
$$

where $w$ is the solution of the adjoint problem

$$
\begin{gathered}
\dot{w}(x, t)=-i \Delta w(x, t)+i a(x) w(x, t) \quad(x \in \Omega, \quad t \geqslant 0), \\
w(x, t)=0 \quad(x \in \partial \Omega, \quad t \geqslant 0), \\
w\left(x, \tau^{*}\right)=\eta(x) \quad(x \in \Omega) .
\end{gathered}
$$

Finally, $u^{*}$ has the bang-bang property

$$
\left\|u^{*}(\cdot, t)\right\|_{L^{2}(\mathcal{O})}=1 \quad\left(t \in\left[0, \tau^{*}\right] \quad \text { a.e. }\right) .
$$


Proof. Note first that it suffices to consider the case $a \geqslant 0$. Indeed, the case of an arbitrary $a \in L^{\infty}(\Omega, \mathbb{R})$ can be reduced to the case $a(x) \geqslant 0$ by an obvious change of variables.

The result is a consequence of Theorem 1.4 and Corollary 1.7, with a particular choice of spaces and operators. More precisely let $X=L^{2}(\Omega), U=L^{2}(\mathcal{O})$ and let the operator $A$ be defined by

$$
\begin{gathered}
\mathcal{D}(A)=H^{2}(\Omega) \cap H_{0}^{1}(\Omega), \\
A \varphi=-i \Delta \varphi+i a(x) \varphi \quad(\varphi \in \mathcal{D}(A)) .
\end{gathered}
$$

The operator $A$ is clearly skew-adjoint and diagonalisable, with eigenvalues of positive imaginary part.

The control operator $B \in \mathcal{L}(U, X)$ is defined by

$$
B u=\chi_{\mathcal{O}} u \quad\left(u \in L^{2}(\mathcal{O})\right) .
$$

We note that, with the above defined spaces of operators, the system (4.6), (4.7) writes in the form $\dot{z}=A z+B u$. Since $A$ is skew-adjoint, in order to apply Theorem 1.4, it suffices to check that the pair $(A, B)$ is exactly controllable in any time $\tau>0$ (see also Remark 1.5). Under the first assumption of the present proposition, this fact follows from Burq [5], combined with classical arguments (see, for instance, Remark 7.4.4 and Theorem 6.7.2 from [29]). Under the second assumption in the present proposition, the exact controllability in any time of $(A, B)$ has been proved in Jaffard [12] and Komornik [13]. Consequently, we can apply Theorem 1.4, so that we obtain (4.8).

In order to apply Corollary 1.7 , we have to check that $\left(A^{*}, B^{*}\right)$ is approximatively observable from any set of positive measure $e \subset\left[0, \tau^{*}\right]$. In PDE terms this means that we have to show that if a solution of (4.9) is such that

$$
w(x, t)=0 \quad(x \in \mathcal{O}, t \in e),
$$

then

$$
w(x, t)=0 \quad(x \in \Omega, t \in \mathbb{R}) .
$$

To accomplish this goal, we note that, due to the fact that $a \in L^{\infty}(\Omega)$, the operator $A$ in (4.13), (4.14) writes $A=i A_{0}$ with $A_{0}$ self-adjoint, diagonalisable and with a spectrum bounded from below. Hence, we can apply Lemma 4.3 to obtain that $w(x, t)=0$ for $x \in \mathcal{O}$ and $t \in \mathbb{R}$. Consequently, using again the exact controllability in any time of the pair $(A, B)$ (thus the exact observability in any time of $\left(A^{*}, B^{*}\right)$ ), it follows that $w(x, t)=0$ for $x \in \Omega$ and $t \in \mathbb{R}$. Finally, we can apply Corollary 1.7 to obtain (4.12).

\section{$5 \quad$ An extension to unbounded control operators}

Some of the results in the previous section can be generalized to the case of unbounded control operators. We give in this section an extension of the maximum principle in Theorem 1.4 and of its consequences to a class of possibly unbounded control operators. The main difficulty in this case arises when we want to apply these results to given systems. More precisely, unlike in Proposition 2.1, the fact that there exists controls which are $L^{2}$ with respect to time and which steer any initial state to any other state in time $\tau$, no longer implies that the same action can be accomplished by controls which are in $L^{\infty}$ with respect to the time variable. Therefore the existence of such controls has to be imposed as an explicit assumption in the statement of the extension to the unbounded case of Theorem 1.4. The main difficulty arises when we want to provide examples of systems with unbounded control operator satisfying this assumption. We provide such an example by constructing an abstract infinite dimensional system with lacunary spectrum in the sense of Zygmund. 
To state the main results in this section we need some notation and some background on unbounded control and observation operators (we refer to [29] for a detailed presentation of these concepts and of related results). As previously, $X, U$ and $Y$ are complex Hilbert spaces, identified with their duals and $\mathbb{T}=\left(\mathbb{T}_{t}\right)_{t \geqslant 0}$ is a strongly continuous semigroup on $X$ generated by an operator $A: \mathcal{D}(A) \rightarrow X$ with resolvent set $\varrho(A)$. The notation $X_{1}$ stands for $\mathcal{D}(A)$ equipped with the norm $\|z\|_{1}:=\|(\beta I-A) z\|$, where $\beta \in \varrho(A)$ is fixed, while $X_{-1}$ is the completion of $X$ with respect to the norm $\|z\|_{-1}:=$ $\left\|(\beta I-A)^{-1} z\right\|$. We use the notation $A$ and $\mathbb{T}$ also for the extensions of the original generator to $X$ and of the original semigroup to $X_{-1}$. It is known that $X_{-1}$ is the dual of $\mathcal{D}\left(A^{*}\right)$ with respect to the pivot space $X$. The semigroup $\mathbb{T}$ can be extended to $X_{-1}$, and then its generator is an extension of $A$, defined on $X$.

In this case the input to state map $\Phi_{t}$ if a linear operator from $L^{2}([0, t], U)$ to $X_{-1}$. As previously, we define for every $t \geqslant 0$ and $u \in L^{2}([0, t], U)$,

$$
\Phi_{t} u=\int_{0}^{t} \mathbb{T}_{t-\sigma} B u(t) \mathrm{d} \sigma .
$$

We say that the control operator $B$ is admissible for the semigroup $\mathbb{T}$ if there exist $t>0$ such that $\operatorname{Ran} \Phi_{t} \subset X$. Notice that if $B$ is an admissible control operator then for every $t \geqslant 0$, we have $\Phi_{t} \in \mathcal{L}\left(L^{2}([0, t], U), X\right)$ (see for instance [29, Proposition 4.2.2]).

In all this section, we assume that $B$ is an admissible control operator for $\mathbb{T}$. In this case, as in the case of bounded control operators, we say that $(A, B)$ is exactly controllable in time $\tau$ if $\operatorname{Ran} \Phi_{\tau}=X$. Moreover, we denote

$$
R_{t}^{\infty}=\Phi_{t}\left(L^{\infty}([0, t], U)\right) .
$$

Remark 5.1. We have seen in Proposition 2.1 that for bounded control operators, the fact that $(A, B)$ is exactly controllable in time $\tau$ implies that $R_{\tau}^{\infty}=X$. This property does no longer hold, in general, for unbounded control operators (it suffices to consider the example of a $1 D$ wave equation with Dirichlet boundary control).

We also need the concepts of admissible observation operators and of observability for such operators. Let $Y$ be another Hilbert space and let $C \in \mathcal{L}\left(X_{1}, Y\right)$ be an observation operator for the semigroup $\mathbb{T}$. For $\tau>0$ and $e \subset[0, \tau]$ of positive measure we define the initial state to output maps $\Psi_{\tau}$ and $\Psi_{\tau, e}$ as in Section 2 with the difference that these maps are now defined on a smaller space, i.e., we have

$$
\Psi_{\tau}, \quad \Psi_{\tau, e} \in \mathcal{L}\left(X_{1}, L^{2}([0, \tau], Y)\right) .
$$

We consider in this section only admissible observation operators which means operators for which $\Psi_{\tau}$ (thus also $\left.\Psi_{\tau, e}\right)$ can be extended to a map in $\mathcal{L}\left(X, L^{2}([0, \tau], Y)\right)$. We say, as in Definition 2.8, that the pair $(A, C)$ is approximately observable from $e$ (a set of positive measure of $[0, \tau]$ ) if $\operatorname{Ker} \Psi_{\tau, e}=\{0\}$. Moreover, we still denote by $\Psi_{\tau}^{d}$ (respectively $\Psi_{\tau, e}^{d}$ ) the output maps corresponding (respectively the restriction to a set of positive measure $e \subset[0, \tau]$ of these output maps) corresponding to the pair $\left(A^{*}, B^{*}\right)$. Note that $B$ is an admissible control operator for $\mathbb{T}$ iff $B^{*}$ is an admissible observation operator for $\mathbb{T}^{*}$ (see, for, instance [29, Theorem 4.4.3]). Moreover, the duality result given in Proposition 2.9 still holds (in fact the already quoted result from [22, Proposition 2.4] covers this more general case). Let us also note that since, as mentioned in Remark 5.1, the property $R_{\tau}^{\infty}=X$, no longer follows from the exact controllability in time $\tau$ of $(A, B)$, the above mentioned duality results cannot be applied to characterize by duality the property $R_{\tau}^{\infty}=X$. However, we have the following result, which goes back to [30] (see also [22, Proposition 2.5]). 
Proposition 5.2. With the above notation, let $\tau>0$. Then the following two properties are equivalent

1. There exists $K_{\tau}>0$ such that

$$
K_{\tau}\left\|\Psi_{\tau}^{d} \varphi\right\|_{L^{1}([0, \tau], Y)} \geqslant\left\|\mathbb{T}_{\tau}^{*} \varphi\right\|_{X} \quad(\varphi \in X) .
$$

2. $R_{\tau}^{\infty}=X$.

Using the concepts and results recalled above, it is not difficult to adapt the proofs of Theorem 1.4 and of Corollary 1.7 to show that the following assertions hold.

Theorem 5.3. Suppose that $B \in \mathcal{L}\left(U, X_{-1}\right)$ is an admissible control operator for the semigroup $\mathbb{T}$ and that $R_{t}^{\infty}=X$ for every $t>0$. Moreover, we consider $z_{0}, z_{1} \in X, z_{0} \neq z_{1}$ such that there exist $\tau>0$ and $u \in L^{\infty}([0, \tau], U)$ such that (1.4) holds. Then there exists a time optimal control $u^{*}$ steering $z_{0}$ to $z_{1}$ in time $\tau^{*}=\tau^{*}\left(z_{0}, z_{1}\right)$. Moreover, there exists $\eta \in X, \eta \neq 0$, such that

$$
\operatorname{Re}\left\langle\left(\Psi_{\tau^{*}}^{d} \eta\right)\left(\tau^{*}-t\right), u^{*}(t)\right\rangle_{U}=\max _{\mathrm{v} \in U,\|\mathrm{v}\|_{U} \leqslant 1} \operatorname{Re}\left\langle\left(\Psi_{\tau}^{d} \eta\right)\left(\tau^{*}-t\right), \mathrm{v}\right\rangle_{U} \quad\left(t \in\left(0, \tau^{*}\right) \quad \text { a.e. }\right) .
$$

Corollary 5.4. Under the assumptions of Theorem 5.3, assume that the pair $(A, B)$ is approximatively controllable from any $e \subset[0, T]$ of positive measure. Then the time optimal control $u^{*}$ is bang-bang, in the sense that

$$
\left\|u^{*}(t)\right\|_{U}=1 \quad\left(t \in\left[0, \tau^{*}\right] \text { a.e. }\right) .
$$

Moreover, the time optimal control is unique.

Remark 5.5. Assuming that $U=\mathbb{C}$, the result in Theorem 5.3 can be easily adapted to other constraints on the control. An example which will be useful in the remaining part of this section is given by constraints of the form

$$
u(t) \in \mathcal{P} \quad(t \geqslant 0)
$$

where $\mathcal{P}$ is the unit square

$$
\mathcal{P}=\{z \in \mathbb{C}|| \operatorname{Re} z|\leqslant 1,| \operatorname{Im} z \mid \leqslant 1\} .
$$

In this case, the maximum principle (5.3) writes

$$
\operatorname{Re}\left[\left(\Psi_{\tau^{*}}^{d} \eta\right)\left(\tau^{*}-t\right) \overline{u^{*}}(t)\right]=\max _{\mathrm{v} \in \mathcal{P}} \operatorname{Re}\left[\left(\Psi_{\tau}^{d} \eta\right)\left(\tau^{*}-t\right) \overline{\mathrm{v}}\right] \quad\left(t \in\left(0, \tau^{*}\right) \quad \text { a.e. }\right) .
$$

We found no example of application of Theorem 5.3 and of Corollary 5.4 coming from PDE's with boundary control. The main difficulty in exhibiting such an example consists in checking the condition $R_{t}^{\infty}=X$ for every $t>0$. To show that the domain of application of the last two results is not reduced to bounded control operators we constructed, in an abstract functional analytic setting, a system (of Schrödinger type) with unbounded control operator for which all the assumptions in Theorem 5.3 hold.

Take $X=\ell^{2}(\mathbb{N}, \mathbb{C})$ and let $A: \mathcal{D}(A) \rightarrow X$ be defined by

$$
(A z)_{k}=i \lambda_{k} z_{k}, \quad \mathcal{D}(A)=\left\{\left.z \in \ell^{2}(\mathbb{N}, \mathbb{C})\left|\sum_{k \in \mathbb{N}} \lambda_{k}^{2}\right| z_{k}\right|^{2}<\infty\right\}
$$

where $\left(\lambda_{k}\right)_{k \in \mathbb{N}}$ is a sequence of positive numbers.

In other words, $A$ is a diagonal operator with the sequence of eigenvectors $\left(e_{k}\right)$ which is the standard 
basis of $\ell^{2}(\mathbb{N}, \mathbb{C})$ and with the corresponding sequence of eigenvalues $\left(i \lambda_{k}\right)$. This fact implies that the adjoint $A^{*}$ of $A$ is given by

$$
\left(A^{*} z\right)_{k}=-i \lambda_{k} z_{k}, \quad \mathcal{D}\left(A^{*}\right)=\mathcal{D}(A) .
$$

Moreover, the semigroups $\mathbb{T}$ (respectively $\mathbb{T}^{*}$ ) generated by $A$ (respectively by $A^{*}$ ) is given by

$$
\left(\mathbb{T}_{t} z\right)_{k}=e^{i \lambda_{k} t} z_{k}, \quad\left(\mathbb{T}_{t}^{*} z\right)_{k}=e^{-i \lambda_{k} t} z_{k}, \quad\left(t \geqslant 0, z \in \ell^{2}(\mathbb{N}, \mathbb{C}), \quad k \in \mathbb{N}\right) .
$$

It is not difficult to check that in this case the space $X_{-1}$ defined at the beginning of this section becomes

$$
X_{-1}=\left\{z=\left(z_{k}\right) \mid \sum_{k \in \mathbb{N}} \frac{\left|z_{k}\right|^{2}}{\lambda_{k}^{2}}<\infty\right\} .
$$

Moreover, the norms on $X_{1}$ and $X_{-1}$ are respectively defined by

$$
\begin{aligned}
& \|z\|_{1}^{2}=\sum_{k \in \mathbb{N}} \lambda_{k}^{2}\left|z_{k}\right|^{2} \quad\left(z \in X_{1}\right), \\
& \|z\|_{-1}^{2}=\sum_{k \in \mathbb{N}} \frac{\left|z_{k}\right|^{2}}{\lambda_{k}^{2}} \quad\left(z \in X_{-1}\right) .
\end{aligned}
$$

Proposition 5.6. Let $X=\ell^{2}(\mathbb{N}, \mathbb{C})$ and let $A$ be the operator defined by (5.6). Assume that the sequence of positive numbers $\left(\lambda_{k}\right)$ in (5.6) is increasing and that

$$
\lim _{k \rightarrow \infty}\left(\lambda_{k+1}-\lambda_{k}\right)=\infty .
$$

Let $B$ be the linear operator associating to each $\mathrm{v} \in \mathbb{C}$ the sequence $B \mathrm{v}$ defined by

$$
(B \mathrm{v})_{k}=\mathrm{v} \quad(\mathrm{v} \in \mathbb{C}, k \in \mathbb{N}) .
$$

Then $B \in \mathcal{L}\left(\mathbb{C}, X_{-1}\right)$ and $B$ is an admissible control operator for the semigroup $\mathbb{T}$ generated by $A$. Moreover, the pair $(A, B)$ is exactly controllable in any time $\tau>0$.

Proof. From (5.10) and the fact that $\left(\lambda_{k}\right)$ is increasing it follows that there exists $\gamma>0$ such that

$$
\left|\lambda_{k+1}-\lambda_{k}\right| \geqslant \gamma \quad(k \in \mathbb{N}) .
$$

Consequently, we have

$$
\sum_{k \in \mathbb{N}} \frac{1}{\lambda_{k}^{2}}<\infty
$$

which, according to the definition of the norm in $X_{-1}$, clearly implies that $B \in \mathcal{L}\left(U, X_{-1}\right)$. Using (5.11) it is easily checked that the operator $B^{*} \in \mathcal{L}\left(X_{1}, \mathbb{C}\right)$ satisfies

$$
B^{*} e_{k}=1 \quad(k \in \mathbb{N}) .
$$

The last formula and (5.10) imply, using the facts that $\left(e_{k}\right)$ is an orthonormal basis of $\ell^{2}(\mathbb{N}, \mathbb{C})$ formed of eigenvectors of $A^{*}$, with corresponding eigenvalues $\left(i \lambda_{k}\right)$, together with the results in $[29$, Proposition 8.1.3] that $B^{*}$ is is an admissible observation operator for $\mathbb{T}^{*}$ and that the pair $\left(A^{*}, B^{*}\right)$ is exactly observable in any time $\tau>0$. From a classical duality result (see, for instance, Theorem 4.4.3 in [29]) it follows that indeed $B$ is an admissible control operator for $\mathbb{T}$. To show that $(A, B)$ is 
exactly controllable in any time $\tau>0$ we first note that condition (5.10) implies, by using a version of Ingham's inequality (see, for instance, [14, Theorem 4.6]) that for every $\tau>0$ there exists $K_{\tau}>0$ such that

$$
K_{\tau}^{2} \int_{0}^{\tau}\left|\sum_{k \in \mathbb{N}} z_{k} e^{-i \lambda_{k} t}\right|^{2} \mathrm{~d} t \geqslant \sum_{k \in \mathbb{N}}\left|z_{k}\right|^{2} \quad\left(z \in \ell^{2}(\mathbb{N}, \mathbb{C})\right) .
$$

Using (5.8) and (5.12) the last formula writes

$$
K_{\tau}^{2} \int_{0}^{\tau}\left|B^{*} \mathbb{T}_{t}^{*} z\right|^{2} \mathrm{~d} t \geqslant\|z\|^{2} \quad\left(z \in X_{1}\right)
$$

i.e., that $\left(A^{*} B^{*}\right)$ is exactly observable in any time $\tau>0$. Using again duality (see, for instance Theorem 11.2.1 in [29]), it follows that indeed $(A, B)$ is exactly controllable in any time $\tau>0$.

We are unable to prove or to disapprove that the assumptions in Proposition 5.6 imply the property $R_{t}^{\infty}=X$, which would be needed in order to apply Theorem 5.3. However, by assuming that the sequence $\left(\lambda_{k}\right)$ is lacunary in the sense of Zygmund, which is a gap condition much stronger then (5.10), this property holds true.

Proposition 5.7. Let $X, A$ and $B$ be the spaces and operators considered in Proposition 5.6. Moreover suppose that, besides the assumptions in Proposition 5.6, the sequence $\left(\lambda_{k}\right)$ is formed of integers and it is lacunary in the sense of Zygmund, i.e. that

$$
\sup _{r \in \mathbb{Z}^{*}} \operatorname{card}\left\{(k, l) \in \mathbb{N}^{2}, \lambda_{k}-\lambda_{l}=r\right\}<+\infty .
$$

Then the corresponding space $R_{t}^{\infty}$, defined in (5.1), coincides with $X$ for every $t>0$.

Proof. Denote

$$
f(s)=\sum_{k \in \mathbb{N}} e^{-i \lambda_{k} s} z_{k} \quad\left(s \geqslant 0, z \in \ell^{2}(\mathbb{N}, \mathbb{C})\right) .
$$

The estimates which follow being uniform with respect to $z \in \ell^{2}(\mathbb{N}, \mathbb{C})$, we do not write explicitly the dependence of $f$ on $z$. Without loss of generality, we can assume that $t \in(0,2 \pi)$. Let is first note that inequality (5.13) in the proof of Proposition 5.6 can be rephrased to assert, for each $t>0$, the existence of a positive constant $K_{t}$ such that

$$
K_{t}^{2} \int_{0}^{t}|f(s)|^{2} \mathrm{~d} s \geqslant \sum_{k \in \mathbb{N}}\left|z_{k}\right|^{2} .
$$

Let

$$
E_{t}=\left\{s \in[0, t],|f(s)| \geqslant \frac{2}{t}\|f\|_{L^{1}[0, t]}\right\} \quad(t \in(0,2 \pi)) .
$$

We clearly have that the Lebesgue measure $\mu\left(E_{t}\right)$ of $E_{t}$ satisfies the condition $\mu\left(E_{t}\right) \leqslant \frac{t}{2}$ for every $t \in(0,2 \pi))$. Hence

$$
\mu\left([0,2 \pi] \backslash E_{t}\right) \geqslant \frac{t}{2} .
$$

At this point we use the key estimate of the proof, which consists in using a quantitative version of a theorem of Zygmund (see Nazarov [24, Theorem III]). According to this result, for every $\varepsilon>0$ there exits $C(\varepsilon)>0$ such that

$$
\|f\|_{L^{2}[0,2 \pi]}^{2} \leqslant \exp \left(\frac{C(\varepsilon)}{\mu\left([0,2 \pi] \backslash E_{t}\right)^{2+\varepsilon}}\right) \int_{[0,2 \pi] \backslash E_{t}}|f(s)|^{2} \mathrm{~d} s .
$$


Using the fact that $|f(s)| \leqslant \frac{2}{t}\|f\|_{L^{1}[0, t]}$ for $s \in[0,2 \pi] \backslash E_{t}$, together with (5.17), we can conclude that

$$
\|f\|_{L^{2}[0, t]}^{2} \leqslant \exp \left(\frac{\widetilde{C}(\varepsilon)}{t^{2+\varepsilon}}\right) \frac{8 \pi}{t^{2}}\|f\|_{L^{1}[0, t]}^{2} \quad(t \in(0,2 \pi)) .
$$

By combining the last estimate and (5.16) it follows that for every $t>0$ there exists $\widetilde{K}_{t}>0$ such that

$$
\widetilde{K}_{t} \int_{0}^{\tau}|f(s)| \mathrm{d} s \geqslant\left(\sum_{k \in \mathbb{N}}\left|z_{k}\right|^{2}\right)^{\frac{1}{2}}
$$

By combining the second formula in (5.8) with the fact that

$$
B^{*} z=\sum_{k \in \mathbb{N}} z_{k} \quad\left(z \in X_{1}\right)
$$

it follows that (5.18) can be rephrased to say that for every $t>0$ there exists $\widetilde{K}_{t}>0$ with

$$
\|z\|_{X} \leqslant \widetilde{K}_{t} \int_{0}^{t}\left|B^{*} \mathbb{T}_{\sigma}^{*} z\right| \mathrm{d} \sigma \quad\left(t>0, z \in X_{1}\right) .
$$

The last estimate implies, by using Proposition 5.2, that indeed $R_{t}^{\infty}=X$ for every $t>0$.

We are now in a position to study the time optimal control problem associated to the system considered in Proposition 5.7.

Proposition 5.8. Let $X, A$ and $B$ be the spaces and operators considered in Proposition 5.7. Then for every $z_{0}, z_{1} \in X, z_{0} \neq z_{1}$ there exists a unique time optimal control $u^{*}$, steering $z_{0}$ to $z_{1}$ in time $\tau^{*}=\tau^{*}\left(z_{0}, z_{1}\right)$, with $\left\|u^{*}\right\|_{L^{\infty}\left(\left[0, \tau^{*}\right], \mathbb{C}\right)} \leqslant 1$. Moreover, there exists $\eta \in X \backslash\{0\}$ such that

$$
u^{*}(t)=\frac{\sum_{n \in \mathbb{N}} \exp \left(i \lambda_{n}\left(\tau^{*}-t\right)\right) \overline{\eta_{n}}}{\left|\sum_{n \in \mathbb{N}} \exp \left(i \lambda_{n}\left(\tau^{*}-t\right)\right) \overline{\eta_{n}}\right|} \quad\left(t \in\left[0, \tau^{*}\right] \quad \text { a.e. }\right) .
$$

Proof. Proposition 5.7 ensure that $R_{t}^{\infty}=X$ for every $t>0$. On the other hand, it is not difficult to check that the proof of Proposition 2.2 adapts to the case of an unbounded admissible $B$ (the main change would occur in the proof of Lemma 2.3, where $\left\|B^{*}\right\|_{\mathcal{L}(X, U)}$ should be replaced by $\left.\left\|\Psi_{\tau}^{d}\right\|_{\mathcal{L}\left(X, L^{2}([0, \tau], U)\right)}\right)$. These facts imply for every $z_{0}, z_{1} \in X$ there exists $T>0$ and a control $u \in L^{\infty}([0, T], \mathbb{C})$ such that the solution $z$ of $(1.1)$ satisfies the condition $z(T)=z_{1}$. Consequently, the pair $(A, B)$ satisfies all the assumptions in Theorem 5.3. Applying this result it follows that there exist a minimal time $\tau^{*}=\tau^{*}\left(z_{0}, z_{1}\right)>0$ and a corresponding control

$$
u^{*} \in L^{\infty}\left(\left[0, \tau^{*}\right], U\right), \quad\left\|u^{*}\right\|_{L^{\infty}\left(\left[0, \tau^{*}\right], U\right)} \leqslant 1,
$$

such that $z_{1}=\mathbb{T}_{\tau^{*}} z_{0}+\Phi_{\tau}^{*} u^{*}$. In addition, there exists $\eta \in X \backslash\{0\}$ such that

$$
\operatorname{Re}\left[\left(\Psi_{\tau^{*}}^{d} \eta\right)\left(\tau^{*}-t\right) \overline{u^{*}(t)}\right]=\max _{\substack{\mathrm{v} \in \mathbb{C} \\|\mathrm{v}| \leqslant 1}} \operatorname{Re}\left[\left(\Psi_{\tau^{*}}^{d} \eta\right)\left(\tau^{*}-t\right) \overline{\mathrm{v}}\right] \quad\left(t \in\left[0, \tau^{*}\right] \quad \text { a.e. }\right) .
$$

On the other hand, from the second formula in (5.8) and (5.12) we see that

$$
\left(\Psi_{\tau^{*}}^{d} \eta\right)\left(\tau^{*}-t\right)=\sum_{n \in \mathbb{N}} \exp \left(-i \lambda_{n}\left(\tau^{*}-t\right)\right) \eta_{n} \quad\left(t \in\left[0, \tau^{*}\right]\right)
$$


where the sum of the above series is computed in $L^{2}([0, T], \mathbb{C})$. Moreover, note that, since $\eta \neq 0$, we can apply Lemma 4.2 to say that

$$
\sum_{n \in \mathbb{N}} \exp \left(-i \lambda_{n}\left(\tau^{*}-t\right)\right) \eta_{n} \neq 0 \quad\left(t \in\left[0, \tau^{*}\right] \text { a.e. }\right) .
$$

Consequently, the combination of (5.20), (5.21) and (5.22) leads to (5.19).

Remark 5.9. Assumption (5.15), appearing in Proposition 5.7 (and consequently in Proposition 5.8) is not satisfied if $\left(\lambda_{n}\right)$ are the eigenvalues of the one dimension Dirichlet Laplacian. Indeed, in this case, up to a multiplicative constant, we have $\lambda_{n}=n^{2}$ for every $n \in \mathbb{N}$. Indeed, it is not difficult to check that the number of solutions $(k, l) \in \mathbb{N}^{2}$ of

$$
k^{2}-l^{2}=2^{m},
$$

tends to $+\infty$ when $m \rightarrow \infty$.

An example of sequence $\left(\lambda_{n}\right)$ satisfying (5.15) is $\lambda_{n}=2^{n}$ for every $n \in \mathbb{N}$. Indeed, it can be easily checked that

$$
\sup _{r \in \mathbb{Z}^{*}} \operatorname{card}\left\{(k, l) \in \mathbb{N}^{2}, 2^{k}-2^{l}=r\right\}=1 .
$$

The constraint $|u(t)| \leqslant 1$ is not appropriate for considering questions such as the switching locus. Therefore, in order to investigate this issue, we have to consider different constraints on the control, such as the one introduced in Remark 5.5. In this case we have:

Proposition 5.10. Let $X, A$ and $B$ be the spaces and operators considered in Proposition 5.7. Consider the system with the control system $(A, B)$ with the control constraints described in Remark 5.5.

Then for every $z_{0}, z_{1} \in X, z_{0} \neq z_{1}$ there exists a time optimal control $u^{*}$, steering $z_{0}$ to $z_{1}$ in time $\tau^{*}=\tau^{*}\left(z_{0}, z_{1}\right)$. Moreover, there exists $\eta \in X, \eta \neq 0$ such that

$$
u^{*}(t)=\operatorname{sign}\left[\operatorname{Re} \sum_{n \in \mathbb{N}} \exp \left(i \lambda_{n}\left(\tau^{*}-t\right)\right) \overline{\eta_{n}}\right]+i \operatorname{sign}\left[\operatorname{Im} \sum_{n \in \mathbb{N}} \exp \left(i \lambda_{n}\left(\tau^{*}-t\right)\right) \overline{\eta_{n}}\right] \quad\left(t \in\left[0, \tau^{*}\right] \text { a.e. }\right)
$$

where, as usual $\operatorname{sign}(a)=-1$ when a is negative, $\operatorname{sign}(a)=1$ for positive $a$ and $\operatorname{sign}(0)=0$.

Proof. We first note that, according to the result in [24, Theorem III] for every $\eta \in X \backslash\{0\}$ we have that

$$
\operatorname{Re} \sum_{n \in \mathbb{N}} \exp \left(i \lambda_{n}\left(\tau^{*}-t\right)\right) \overline{\eta_{n}} \neq 0, \quad \operatorname{Im} \sum_{n \in \mathbb{N}} \exp \left(i \lambda_{n}\left(\tau^{*}-t\right)\right) \overline{\eta_{n}} \neq 0 \quad\left(t \in\left[0, \tau^{*}\right] \text { a.e }\right) .
$$

On the other hand, by combining (5.5) and (5.21), we have that

$\operatorname{Re}\left[\sum_{n \in \mathbb{N}} \exp \left(-i \lambda_{n}\left(\tau^{*}-t\right)\right) \eta_{n} \overline{u^{*}}(t)\right]=\max _{\mathrm{v} \in \mathcal{P}} \operatorname{Re}\left[\sum_{n \in \mathbb{N}} \exp \left(-i \lambda_{n}\left(\tau^{*}-t\right)\right) \eta_{n} \overline{\mathrm{v}}\right] \quad\left(t \in\left(0, \tau^{*}\right) \quad\right.$ a.e. $)$.

The last two formulas clearly imply the conclusion of the proposition.

Remark 5.11. We also note that, under the above assumptions, every input satisfying the maximum principle (5.23) is the time optimal control steering 0 to $\Phi_{\tau^{*}} u^{*}$. The proof of this assertion can be done following line by line the proof given, for the finite dimensional case, in Agrachev and Sachkov [1, Theorem 15.4 ]. 
Corollary 5.12. Let $X, A$ and $B$ be as in Proposition 5.10, let $z_{0}, z_{1} \in X$. Then the time optimal control $u^{*}$, steering $z_{0}$ to $z_{1}$ in time $\tau^{*}=\tau^{*}\left(z_{0}, z_{1}\right)$ is unique and it satisfies

$$
u^{*}(t) \in\{-1-i,-1+i, 1-i, 1+i\} \quad\left(t \in\left[0, \tau^{*}\right] \text { a.e. }\right) .
$$

Moreover, for every $N \in \mathbb{N}$ there exist $z_{0}, z_{1} \in X$ such that $\operatorname{Re} u^{*}$ and $\operatorname{Im} u^{*}$ switch between -1 and 1 more than $N$ times for $t \in\left[0, \tau^{*}\right]$.

Proof. The fact that any time optimal control satisfies (5.25) follows directly from (5.24) and (5.23). The uniqueness of the time optimal control follows next from (5.25) by the usual convexity argument.

To show that the number of switches can be arbitrarily large, we consider an arbitrary $\tau^{*}>0$, we take $m \in \mathbb{N}$, which will be made precise later, and we define

$$
u^{*}(t)=\operatorname{sign}\left[\cos \left(\lambda_{m}\left(\tau^{*}-t\right)\right)\right]+i \operatorname{sign}\left[\sin \left(\lambda_{m}\left(\tau^{*}-t\right)\right)\right] \quad\left(t \in\left[0, \tau^{*}\right]\right) .
$$

The control $u^{*}$ satisfies the maximum principle with $\eta=e_{m}$ (recall that $\left(e_{n}\right)$ is the standard basis in $l^{2}$ so that, according to Remark 5.11, $u^{*}$ is the time optimal control steering 0 to $\Phi_{\tau}^{*} u^{*}$ under the constraint (5.4). Choosing $m$ such that $\left[\frac{\lambda_{m} \tau^{*}}{\pi}\right] \geqslant N$, it is easily checked that the real and the imaginary parts of $u^{*}$ switch from -1 to 1 more than $N$ times.

\section{Concluding remarks and open questions}

Our results in the previous section are, as far as we know, the first establishing the maximum principle and the bang-bang property for the time optimal controls in the case of a class of time reversible systems (of Schrödinger type) with controls localized in a strict subset of the spatial domain. If we take controls active in the whole spatial domain, one can easily adapt our methods to the wave or Euler-Bernoulli plate equation. More generally, the results in the previous can be easily adapted to systems of the form

$$
\ddot{w}+A_{0} w=u,
$$

where $A_{0}$ is a positive operator in a Hilbert space $H$ and $u$ is the input function. We can easily check that the above equation can be written as a first order system satisfying the assumptions in Theorem 1.4 and Corollary 1.7, so that we have the maximum principle and the bang-bang property for the associated time optimal control problem. Since this question has been tackled directly, by quite similar methods, in Fattorini [8] and Krabs [15] we do not give the detailed argument here. Note that, taking $H=L^{2}(\Omega)$, where $\Omega \subset \mathbb{R}^{n}$ is bounded and with a smooth boundary, and $A_{0}$ to be the Dirichlet Laplacian (respectively the square of the Dirichlet Laplacian) in $\Omega$, (6.26) becomes the wave (respectively the Euler-Bernoulli plate) equation, with control active in all of $\Omega$. We also refer to Kunisch and Wachsmuth [16] for a theoretical and numerical study of the time optimal control problem for the wave equation.

An interesting and partially open question consists in obtaining the maximum principle and the bang-bang property of time optimal controls for systems governed by the Euler-Bernoulli plate equation with localized distributed control. The question is challenging, in particular, since most of the control theoretic results known for the Schrödinger equation have a natural counterpart for the EulerBernoulli plate equation (at least for hinged boundary conditions). It turns out that the situation seems unclear in the case of time optimal control problems, at least as far as the bang-bang property is concerned. More precisely, consider the one dimensional case (Euler-Bernoulli beam equation) with locally distributed control. The main technical obstacle (within our method) in obtaining the bangbang property of time optimal controls consists in the fact that, in order to apply Corollary 1.7, one 
would need a counterpart of the uniqueness result in Lemma 4.1, with the sequence $\left( \pm n^{2}\right)_{n \geqslant 1}$ (which is bounded neither from below nor from above) instead of $\left(\lambda_{n}\right)$. Therefore, even in one space dimension, establishing the uniqueness of time optimal controls and their bang-bang property are open questions.

However, we have a partial result, asserting that the maximum principle holds for the time optimal control problem for a system governed by the Euler-Bernoulli plate equation with locally distributed control. More precisely, consider the system

$$
\begin{gathered}
\ddot{z}(x, t)=-\Delta^{2} z(x, t)+\chi_{\mathcal{O}}(x) u(x, t) \quad(x \in \Omega, t \geqslant 0), \\
z(x, t)=\Delta z(x, t)=0 \quad(x \in \partial \Omega, t \geqslant 0),
\end{gathered}
$$

where $\Omega \subset \mathbb{R}^{n}$ is an open set, $\mathcal{O}$ is an open subset of $\Omega, \chi_{\mathcal{O}}$ is the characteristic function of $\mathcal{O}$ and the control $u$ is such that $\|u(\cdot, t)\|_{L^{2}(\mathcal{O})} \leqslant 1$ for almost every $t \geqslant 0$.

Proposition 6.1. Assume that one of the assumptions

1. The open set $\Omega$ is bounded, $\partial \Omega$ is of class $C^{\infty}$ and $\mathcal{O}$ satisfies the geometric optics condition;

2. The open set $\Omega$ is a rectangular domain and $\mathcal{O}$ is an arbitrary nonempty open subset of $\Omega$,

holds. Then for every $f_{0}, f_{1} \in H^{2}(\Omega) \cap H_{0}^{1}(\Omega)$ and $g_{0}, g_{1} \in L^{2}(\Omega)$, with $\left(f_{0}, g_{0}\right) \neq\left(f_{1}, g_{1}\right)$, there exists a time optimal control $u^{*}$ steering the solution of (6.27)-(6.28) from $\left(f_{0}, g_{0}\right)$ (at $\left.t=0\right)$ to $\left(f_{1}, g_{1}\right)$ (at $\left.t=\tau^{*}\right)$. Moreover, there exists $\left(\eta_{0}, \eta_{1}\right) \in\left[H^{2}(\Omega) \cap H_{0}^{1}(\Omega)\right] \times L^{2}(\Omega)$, with $\left(\eta_{0}, \eta_{1}\right) \neq(0,0)$ such that

$$
\int_{\mathcal{O}} \dot{w}(x, t) \overline{u^{*}}(x, t) \mathrm{d} x=\max _{\substack{\mathrm{v} \in L^{2}(\mathcal{O}),\|\mathrm{v}\|_{L^{2}(\mathcal{O})} \leqslant 1}} \int_{\mathcal{O}} \dot{w}(x, t) \overline{\mathrm{v}}(x) \mathrm{d} x,
$$

where $w$ is the solution of the adjoint problem,

$$
\begin{array}{ccc}
\ddot{w}(x, t)=-\Delta^{2} w(x, t) & (x \in \Omega, & t \in \mathbb{R}), \\
w(x, t)=\Delta w(x, t)=0 & (x \in \partial \Omega, & t \in \mathbb{R}), \\
w\left(x, \tau^{*}\right)=\eta_{0}(x), \dot{w}\left(x, \tau^{*}\right)=\eta_{1}(x) & (x \in \Omega) .
\end{array}
$$

Proof. The proof follows closely the reasoning used in the first part of the proof of Proposition 4.4. More precisely, it suffices to apply Theorem 1.4, with a particular choice of spaces and operators. Take $X=\left[H^{2}(\Omega) \cap H_{0}^{1}(\Omega)\right] \times L^{2}(\Omega), U=L^{2}(\mathcal{O})$ and consider the operator $A$ defined by

$$
\begin{gathered}
\mathcal{D}(A)=\left\{f \in H^{4}(\Omega) \cap H_{0}^{1}(\Omega), \Delta f=0 \text { on } \partial \Omega\right\} \times\left(H^{2}(\Omega) \cap H_{0}^{1}(\Omega)\right), \\
A\left[\begin{array}{l}
f \\
g
\end{array}\right]=\left[\begin{array}{cc}
0 & I \\
-\Delta^{2} & 0
\end{array}\right]\left[\begin{array}{l}
f \\
g
\end{array}\right]=\left[\begin{array}{c}
g \\
-\Delta^{2} f
\end{array}\right] \quad\left(\left[\begin{array}{l}
f \\
g
\end{array}\right] \in \mathcal{D}(A)\right) .
\end{gathered}
$$

The control operator $B \in \mathcal{L}(U, X)$ is defined by

$$
B u=\left[\begin{array}{c}
0 \\
\chi_{\mathcal{O}} u
\end{array}\right] \quad\left(u \in L^{2}(\mathcal{O})\right) .
$$

We note that, with the above defined spaces of operators, the system (6.27), (6.28) writes in the form $\dot{Z}=A Z+B u$, where $Z=\left[\begin{array}{l}z \\ \dot{z}\end{array}\right]$.

In order to apply Theorem 1.4, it suffices to check that the pair $(A, B)$ is exactly controllable in any time $\tau>0$. This fact is classical and follows from known (already used above) exact controllability results for the Schrödinger equation (see, for instance, [29, Proposition 7.5.1] or [18, Section 5]). Consequently, we can apply Theorem 1.4, so that we obtain (6.29). 
Besides the theoretical open questions raised above, the numerical analysis of the time optimal control problem for infinite dimensional systems is an essentially open field, at least for point targets. An interesting track to tackle these issues could consist in adapting some of the results on the numerical approximation of norm optimal controls (see, for instance, Ervedoza and Zuazua [6] and references therein).

\section{References}

[1] A. A. Agrachev and Y. L. Sachkov, Control theory from the geometric viewpoint, vol. 87 of Encyclopaedia of Mathematical Sciences, Springer-Verlag, Berlin, 2004. Control Theory and Optimization, II.

[2] V. Barbu and T. Precupanu, Convexity and optimization in Banach spaces, vol. 10 of Mathematics and its Applications (East European Series), D. Reidel Publishing Co., Dordrecht, romanian ed., 1986.

[3] C. Bardos, G. Lebeau, And J. Rauch, Sharp sufficient conditions for the observation, control and stabilization of waves from the boundary, SIAM J. Control. and Optim., 30 (1992), pp. 10241065.

[4] R. Bellman, I. Glicksberg, and O. Gross, On the "bang-bang" control problem, Quart. Appl. Math., 14 (1956), pp. 11-18.

[5] N. Burq, Contrôlabilité exacte des ondes dans des ouverts peu réguliers, Asymptot. Anal., 14 (1997), pp. 157-191.

[6] S. Ervedoza And E. Zuazua, The wave equation: Control and numerics, in Control of Partial Differential Equations, Lecture Notes in Mathematics, CIME Subseries, 2012. to appear.

[7] H. O. Fattorini, Time-optimal control of solutions of operational differenital equations, J. Soc. Indust. Appl. Math. Ser. A Control, 2 (1964), pp. 54-59.

[8] — The time optimal problem for distributed control of systems described by the wave equation, in Control theory of systems governed by partial differential equations (Conf. Naval Surface Weapons Center, Silver Spring, Md., 1976), Academic Press, New York, 1977, pp. 151-175.

[9] _ Infinite Dimensional Linear Control Systems. The Time Optimal and Norm Optimal Control Problems, North-Holland Mathematics Studies, 201, Elsevier, Amsterdam, 2005.

[10] F. Guo, K. Guo, And C. Zhang, Lack of uniformly exponential stabilization for isometric $C_{0}{ }^{-}$ semigroups under compact perturbation of the generators in Banach spaces, Proc. Amer. Math. Soc., 135 (2007), pp. 1881-1887.

[11] F. M. Hante, M. Sigalotti, and M. Tucsnak, On conditions for asymptotic stability of dissipative infinite-dimensional systems with intermittent damping, J. Differential Equations, 252 (2012), pp. 5569-5593.

[12] S. Jaffard, Contrôle interne exact des vibrations d'une plaque carrée, C. R. Acad. Sci. Paris Sér. I Math., 307 (1988), pp. 759-762. 
[13] V. Komornik, On the exact internal controllability of a Petrowsky system, J. Math. Pures Appl. (9), 71 (1992), pp. 331-342.

[14] V. Komornik And P. Loreti, Fourier series in control theory, Springer Monographs in Mathematics, Springer-Verlag, New York, 2005.

[15] W. Krabs, On time-minimal distributed control of vibrations, Appl. Math. Optim., 19 (1989), pp. $65-73$.

[16] K. Kunisch And D. Wachsmuth, Time optimal control of the wave equation, its regularization and numerical realization, SFB-Report, 2011-017 (2011), pp. 1203-1215.

[17] K. Kunisch And L. Wang, Time optimal control of the heat equation with pointwise control constraints, preprint, (2011).

[18] G. Lebeau, Contrôle de l'équation de Schrödinger, J. Math. Pures Appl. (9), 71 (1992), pp. 267291.

[19] E. B. Lee And L. Markus, Foundations of optimal control theory, John Wiley \& Sons Inc., New York, 1967.

[20] J.-L. Lions, Contrôle optimal de systèmes gouvernés par des équations aux dérivées partielles, Avant propos de P. Lelong, Dunod, Paris, 1968.

[21] K. LIU, Locally distributed control and damping for the conservative systems, SIAM J. Control Optim., 35 (1997), pp. 1574-1590.

[22] S. Micu, I. Roventa, And M. Tucsnak, Time optimal boundary controls for the heat equation, J. Funct. Anal., 263 (2012), pp. 25-49.

[23] V. J. Mizel and T. I. Seidman, An abstract bang-bang principle and time-optimal boundary control of the heat equation, SIAM J. Control Optim., 35 (1997), pp. 1204-1216.

[24] F. L. NAZARov, Local estimates for exponential polynomials and their applications to inequalities of the uncertainty principle type, Algebra i Analiz, 5 (1993), pp. 3-66.

[25] K. D. Phung And G. Wang, An observability for parabolic equations from a measurable set in time, preprint, (2011).

[26] K. D. Phung, G. Wang, and X. Zhang, On the existence of time optimal controls for linear evolution equations, Discrete Contin. Dyn. Syst. Ser. B, 8 (2007), pp. 925-941 (electronic).

[27] F. Reifler And A. Vogt, Unique continuation of some dispersive waves, Comm. Partial Differential Equations, 19 (1994), pp. 1203-1215.

[28] E. C. Titchmarsh, The theory of functions, Oxford University Press, London, second ed., 1939.

[29] M. TucSnaK And G. Weiss, Observation and control for operator semigroups, Birkhäuser Advanced Texts: Basler Lehrbücher. [Birkhäuser Advanced Texts: Basel Textbooks], Birkhäuser Verlag, Basel, 2009.

[30] G. WANG, $L^{\infty}$-null controllability for the heat equation and its consequences for the time optimal control problem, SIAM J. Control Optim., 47 (2008), pp. 1701-1720. 
[31] J. ZABCZYK, Mathematical control theory: an introduction, Systems \& Control: Foundations \& Applications, Birkhäuser Boston Inc., Boston, MA, 1992.

[32] A. Zygmund, Trigonometric series. 2nd ed. Vols. I, II, Cambridge University Press, New York, 1959. 